DOI: $10.17516 / 1997-1397-2020-13-6-792-796$

УДК 539.374

\title{
New Classes of Solutions of Dynamical Problems of Plasticity
}

\author{
Sergei I. Senashov* \\ Olga V. Gomonova ${ }^{\dagger}$ \\ Irina L. Savostyanova ${ }^{\ddagger}$
}

Department of Economic Information Systems, Reshetnev Siberian State University of Science and Technology,

31 Krasnoyarsky Rabochy Av., Krasnoyarsk, 660037, Russia

Olga N. Cherepanova ${ }^{\S}$

Department of Mathematical Analysis and Differential Equations,

Siberian Federal University,

Svobodny 79, Krasnoyarsk, 660041, Russia

Received 10.05.2020, received in revised form 10.06.2020, accepted 20.10.2020

\begin{abstract}
Dynamical problems of the theory of plasticity have not been adequately studied. Dynamical problems arise in various fields of science and engineering but the complexity of original differential equations does not allow one to construct new exact solutions and to solve boundary value problems correctly. One-dimensional dynamical problems are studied rather well but two-dimensional problems cause major difficulties associated with nonlinearity of the main equations. Application of symmetries to the equations of plasticity allow one to construct some exact solutions. The best known exact solution is the solution obtained by B.D. Annin. It describes non-steady compression of a plastic layer by two rigid plates. This solution is a linear one in spatial variables but includes various functions of time. Symmetries are also considered in this paper. These symmetries allow transforming exact solutions of steady equations into solutions of non-steady equations. The obtained solution contains 5 arbitrary functions.
\end{abstract}

Keywords: differential equation, plasticity, dynamical problem, exact solution, symmetries.

Citation: S.I. Senashov, O.V. Gomonova I.L. Savostyanova, O.N. Cherepanova, New Classes of Solutions of Dynamical Problems of Plasticity, J. Sib. Fed. Univ. Math. Phys., 2020, 13(6), 792-796.

DOI: 10.17516/1997-1397-2020-13-6-792-796.

\section{Introduction}

There is an extensive literature on the theory of plasticity. The reason is that problems considered in this theory are very important for various practical applications. These problems arise in the design of machines and technological processes where plastic deformations are present, in various applications to armaments industry (for example, projectile penetration theory, etc.). Contemporary and classical studies deal mainly with static problems. This is not because dynamical problems are not important but because of lack of progress in developing appropriate

\footnotetext{
*sen@sibsau.ru

†gomonova@sibsau.ru

†ruppa@inbox.ru

$\S$ cheronik@mail.ru

(c) Siberian Federal University. All rights reserved
} 
methods to solve these problems. The spatial solution of dynamical equations was first obtained by B. D. Annin in 1978 [2]. This solution is linear in spatial variables and contains several arbitrary functions that depend on time. The solution was constructed with the use of group of point symmetries admitted by the system of equations of dynamical theory of plasticity. Later, new exact solutions of some plane dynamical problems were constructed,. They are based on group properties of the equations. New solutions of the dynamical equations are given in [8]. They are based on transformation of steady-state solutions into non-steady solutions.

New classes of exact solutions of dynamical problems of the theory of plasticity are proposed in the paper. They contain 5 arbitrary functions.

\section{Problem definition}

Let $x=x_{1}, y=x_{2}, z=x_{3}$ is Cartesian coordinate system, $u=v_{1}, v=v_{2}, w=v_{3}$ are components of strain rate vector, $e_{i j}$ are components of strain velocity tensor, $\sigma_{i j}$ are components of stress tensor. The components of strain velocity tensor and stress tensor satisfy the equations of motion

$$
\frac{d v_{i}}{d t}=\partial_{i} \sigma_{i j}, \quad i, j=1,2,3
$$

Here $\frac{d v_{i}}{d t}=\partial_{t} v_{i}+v_{j} \partial_{j} v_{i}$ is a full or substantial derivative. Einstein summation convention is applied here. Components of the stress deviator tensor and the strain velocity tensor are coaxial

$$
\sigma_{i j}-\delta_{i j} p=\lambda e_{i j}=\lambda\left(\partial_{j} v_{i}+\partial_{i} v_{j}\right) / 2
$$

where, $\delta_{i j}$ is the Kronecker symbol, $\lambda$ is a non-negative function, $3 p=\sigma_{i i}$.

It is assumed that medium is incompressible. Then we have incompressibility equation

$$
\partial_{i} v_{i}=0
$$

In addition to system of equations (1)-(3), von Mises yield criterion is used

$$
\left(\sigma_{11}-p\right)^{2}+\left(\sigma_{22}-p\right)^{2}+\left(\sigma_{33}-p\right)^{2}+2\left(\sigma_{12}^{2}+\sigma_{13}^{2}+\sigma_{23}^{2}\right)=2 k_{s}^{2},
$$

where $k_{s}$ is the shear yield stress.

\section{Group properties of the equations of dynamical theory of plasticity}

Lie group of point symmetries admitted by equations (1)-(4) is described in [3]. It is generated by the following operators

$$
\begin{gathered}
X_{0}=\partial_{i}, \quad M=t \partial_{t}+x_{i} \partial_{x_{i}}, \quad S=\varphi(t) \partial_{p}, \quad T_{i}=f_{i}(t) \partial_{x_{i}}+f_{i}^{\prime}(t) \partial_{v_{i}}-x_{i} f_{i}^{\prime \prime}(t) \partial_{p}, \\
Z_{1}=x_{2} \partial_{x_{3}}-x_{3} \partial_{x_{2}}+v_{2} \partial_{v_{3}}-v_{3} \partial_{v_{2}} .
\end{gathered}
$$

There is no Einstein summation convention in (5). Two more operators $Z_{2}, Z_{3}$ can be obtained from $Z_{1}$ by circular permutation of indices. Functions $\varphi(t), f_{i}(t)$ are arbitrary functions from the class $C^{\infty}$. Therefore, operators (5) generate an infinite Lie algebra. Derivatives with respect to variable $t$ is designated by primes. 
Group properties of differential equations can be used for various purposes. They are most often used to construct invariant solutions - the solutions which do not change with continuous transformations that correspond to the operators of algebra (5). The invariant solutions of the plasticity equations and methods of their construction are described more fully in [2] and in the literature therein. The procedure of deformation of the exact solutions using point symmetries and the reduction of an exact solution into another one in the case of plane steady equations of ideal plasticity were shown [7]. We use the group of point symmetries for transformation of new stationary solutions into new non-stationary ones for the case of three-dimensional plasticity equations. This approach was firstly applied for construction of new solutions in [8].

\section{New stationary solution of system (1)-(4)}

As system (1)-(4) admits the operator $X_{0}=\partial_{t}$, one can find the invariant solutions of this system that do not depend on the variable $t$. These solutions can be determined from the system

$$
\begin{gathered}
v_{j} \partial_{j} v_{i}=\partial_{i} \sigma_{i j}, \quad \sigma_{i j}-\delta_{i j} p=\lambda e_{i j}=\lambda\left(\partial_{j} v_{i}+\partial_{i} v_{j}\right) / 2, \\
\partial_{i} v_{i}=0, \quad\left(\sigma_{11}-p\right)^{2}+\left(\sigma_{22}-p\right)^{2}+\left(\sigma_{33}-p\right)^{2}+2\left(\sigma_{12}^{2}+\sigma_{13}^{2}+\sigma_{23}^{2}\right)=2 k_{s}^{2}
\end{gathered}
$$

System (6) is simpler than the initial one because it has fewer independent variables. Some of solutions of the system are given in [8]. As far as we know, there are no other solutions of the considered system [1-3]. Let us find an invariant solution of system (6) regarding the one-dimensional subalgebra that admits the operator $\frac{1}{\alpha} \partial_{x}+\frac{1}{\beta} \partial_{y}-\frac{2}{\gamma} \partial_{z}$. This solution has the following form

$$
u=A g(\alpha x+\beta y+\gamma z), v=B g(\alpha x+\beta y+\gamma z), w=C g(\alpha x+\beta y+\gamma z), p=F(\alpha x+\beta y+\gamma z) .
$$

Here $A, B, C, \alpha, \beta, \gamma$ are arbitrary constants, and functions $g, F$ are determined from system (6). One can obtain the following relations between the functions and the constants

$$
\begin{gathered}
\alpha A+\beta B+\gamma C=0, \quad F=\frac{1}{2} g^{2}+\delta, \\
\alpha A^{2}+\beta A B+\gamma A C=\alpha, \quad \alpha A B+\beta B^{2}+\gamma B C=\beta, \quad \alpha A B+\beta B C+\gamma C^{2}=\gamma,
\end{gathered}
$$

here $\delta$ is an arbitrary constant. Equalities (7) and (8) imply that all components of the stress tensor are constant and have the form

$$
\begin{gathered}
\sigma_{11}=p+\frac{\alpha A}{D}, \quad \sigma_{22}=p+\frac{\beta B}{D}, \quad \sigma_{33}=p+\frac{\gamma C}{D}, \\
\sigma_{12}=\frac{\beta A+\alpha B}{2 D}, \quad \sigma_{13}=\frac{\gamma A+\alpha C}{2 D}, \quad \sigma_{23}=\frac{\gamma B+\beta C}{2 D}, \\
D^{2}=2 k_{s}^{2}\left((\alpha A)^{2}+(\beta B)^{2}+(\gamma C)^{2}+\frac{1}{2}(\beta A+\alpha B)^{2}+\frac{1}{2}(\gamma A+\alpha C)^{2}+\frac{1}{2}(\gamma B+\beta C)^{2}\right) .
\end{gathered}
$$

The similar solution with the absence of convective terms was constructed in [8].

\section{Deformation of stationary solution of system (1)-(4)}

Here, the stationary solution obtained above with the use of transformations (5) is deformed into non-stationary solution of initial system (1)-(4). For this purpose, a notable property of 
the point symmetries is used, namely, the symmetries transform any exact solution of system (1)-(4) into a new exact solution of this system.

System (1)-(4) admits operators $S=\varphi(t) \partial_{p}, \quad T_{i}=f_{i}(t) \partial_{x_{i}}+f_{i}^{\prime}(t) \partial_{v_{i}}-x_{i} f_{i}^{\prime \prime}(t) \partial_{p}, \quad(i=$ $1,2,3)$. It means that the system is not changed under the following transformations

$$
x_{i}^{\prime}=x_{i}+a_{i} f_{i}(t), \quad v_{i}^{\prime}=v_{i}+a_{i} f_{i}^{\prime}(t), \quad p_{i}^{\prime}=p-\sum_{i=1}^{3} a_{i} x_{i} f_{i}^{\prime \prime}(t)+a_{4} \varphi(t) .
$$

Here variables without primes are initial ones and variables with primes are obtained as a result of point symmetries that correspond to subalgebra generated by the operators $S, T_{i}$. Parameters $a_{i}$ are group parameters which change continuously in neighbourhood of zero $x_{1}=x, x_{2}=y, x_{3}=$ $z$.

Let us assume that $v_{i}^{1}, p^{1}$ is a solution of system (1)-(4). Then, in accordance with $(9), v_{i}^{2}, p^{2}$ of the form

$$
\begin{gathered}
v_{1}^{2}=v_{1}^{1}\left(t, x_{1}+a_{1} f_{1}(t), x_{2}+a_{2} f_{2}(t), x_{3}+a_{3} f_{3}(t)\right)+a_{1} f_{1}^{\prime}(t), \\
v_{2}^{2}=v_{2}^{1}\left(t, x_{1}+a_{1} f_{1}(t), x_{2}+a_{2} f_{2}(t), x_{3}+a_{3} f_{3}(t)\right)+a_{2} f_{2}^{\prime}(t), \\
v_{3}^{2}=v_{3}^{1}\left(t, x_{1}+a_{1} f_{1}(t), x_{2}+a_{2} f_{2}(t), x_{3}+a_{3} f_{3}(t)\right)+a_{3} f_{3}^{\prime}(t), \\
\left.p^{2}=p^{1}\left(t, x_{1}+a_{1} f_{1}(t), x_{2}+a_{2} f_{2}(t), x_{3}+a_{3} f_{3}(t)\right)-\sum_{i=1}^{3} x_{i} f_{i}^{\prime \prime}(t)\right)
\end{gathered}
$$

are also an exact solution of the same system. This property is used to construct new solutions of system (1)-(4). Let us apply formulae (11) to the solution constructed above. Then we obtain

$$
\begin{gathered}
u=A g\left(\alpha\left(x+a_{1} f_{1}(t)\right)+\beta\left(y+a_{2} f_{2}(t)\right)+\gamma\left(z+a_{3} f_{3}(t)\right)+a_{1} f_{1}^{\prime}(t),\right. \\
v=B g\left(\alpha\left(x+a_{1} f_{1}(t)\right)+\beta\left(y+a_{2} f_{2}(t)\right)+\gamma\left(z+a_{3} f_{3}(t)\right)+a_{2} f_{2}^{\prime}(t),\right. \\
w=C g\left(\alpha\left(x+a_{1} f_{1}(t)\right)+\beta\left(y+a_{2} f_{2}(t)\right)+\gamma\left(z+a_{3} f_{3}(t)\right)+a_{3} f_{3}^{\prime}(t),\right. \\
p=\frac{1}{2} g^{2}\left(\alpha\left(x+a_{1} f_{1}(t)\right)+\beta\left(y+a_{2} f_{2}(t)\right)+\gamma\left(z+a_{3} f_{3}(t)\right)-\right. \\
-x a_{1} f_{1}^{\prime \prime}(t)-y a_{2} f_{2}^{\prime \prime}(t)-z a_{3} f_{3}^{\prime \prime}(t)+\varphi(t) .
\end{gathered}
$$

The components of the stress tensor corresponded to the velocity field (12) coincide with (9).

\section{Conclusion}

A non-steady solution containing 5 variable functions was constructed from a stationary solution. The method of construction of non-stationary solutions of dynamical equations of plasticity from a stationary solution was shown in the paper. These solutions can be used for the analysis of technological processes when the stress state is stationary but the process is dynamical.

This work was supported by the Krasnoyarsk Mathematical Center and financed by the Ministry of Science and Higher Education of the Russian Federation in the framework of the 
establishment and development of regional Centers for Mathematics Research and Education (Agreement No. 075-02-2020-1631).

\section{References}

[1] Ivlev D. D. et al. The limiting state of deformable bodies and rocks, Moscow, Physmathlit, 1964 (in Russian).

[2] Annin B. D., Bytev V. O., Senashov S. I. Group properties of equations of elasticity and plasticity, Novosibirsk, Nauka, 1985 (in Russian).

[3] Polyanin A.D., Zaittsev V.F. Handbook of nonlinear partial differential equations, CRC Press, London, New York, Second Edition, 2012.

[4] Novatsky V. K. Wave problems of the theory of plasticity. Moscow, Mir, 1978 (in Russian)

[5] Zadoyan M. A. Space problems of the theory of plasticity. Moscow, Nauka, 1992 (in Russian)

[6] Ishlinky A. Yu., Ivlev D. D. Mathematical theory of plasticity. Moscow, Physmathlit, 2001 (in Russian)

[7] Senashov S.I., Yakhno A.N. Reproduction of solutions for bidimensional ideal plasticity, Journal of Non -Linear Mechanics, 42(2007), 500-503.

[8] Senashov S. I., Savostyanova I. L. New solutions of dynamical equations of plasticity, Journal of Applied and Industrial Mathematics, XXII, 4(80), 2019, 89-94.

\section{Новые классы решений динамических задач пластичности}

Сергей И. Сенашов

Ольга В. Гомонова

Ирина Л. Савостьянова

Сибирский государственный университет науки и технологий им. Решетнева,

Красноярский рабочий 31, Красноярск, 660037, Россия

Ольга Н.Черепанова

Сибирский федеральный университет, Свободный 79, Красноярск, 660041, Россия

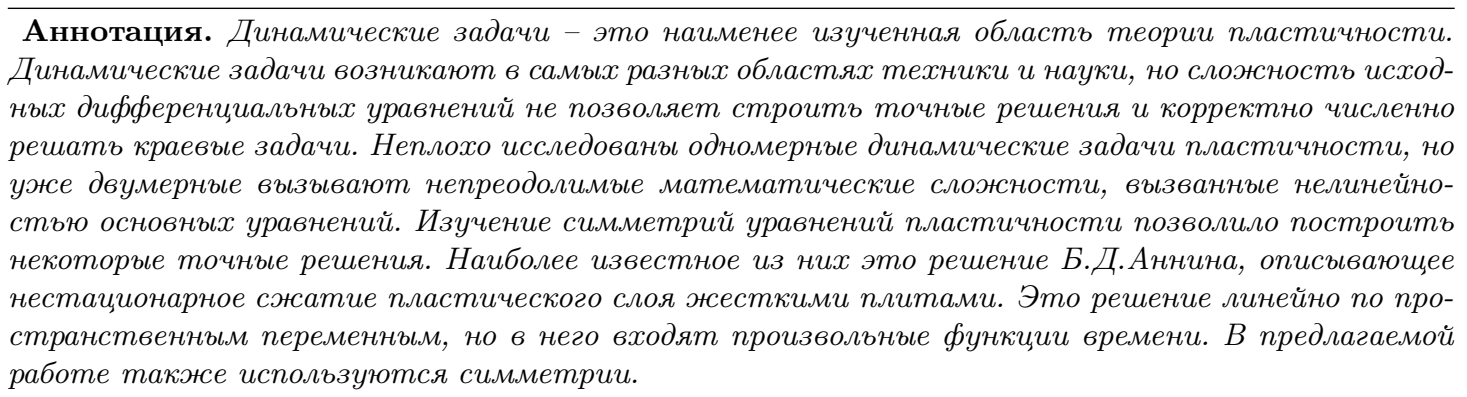
Ключевые слова: дифференциальные уравнения, пластичность, динамические задачи, точные решения, симметрии. 